\title{
Auxiliary Dipoles to Compensate for the Finite Size of the Planar Scanning Area in Near-to-Far-Field Transformations
}

\author{
Vladimir Volski, Member, IEEE, Sen Yan, Guy A. E. Vandenbosch, Fellow, IEEE, Tim Claeys, Davy \\ Pissoort, Senior Member, IEEE
}

\begin{abstract}
A novel algorithm to obtain an accurate representation of the far-field emissions starting from a finite planar scan of the magnetic near-field is proposed. The algorithm introduces so-called auxiliary sources that compensate for the finite size of the scan area. While previous works typically introduce equivalent sources to represent the fields inside the scanning area, the auxiliary sources introduced in this paper approximate the fields outside of the scanning area. The auxiliary sources are located within the pcb under the scanning area at the locations where the scanned field strengths are above a pre-chosen threshold. Their complex values are chosen such that they accurately represent the fields at the edge of the scanning area. Several numerical examples show that the application of these auxiliary sources considerably improves the accuracy of the obtained far-field.
\end{abstract}

Index Terms-Near Field, Equivalence Principle, Radiation pattern.

\section{I ITroduction}

M EASUREMENT procedures to simultaneously predict the level of radiated fields emitted by an electronic device and identify the location of the sources of unwanted emissions are of great interest in the Electro-Magnetic Compatibility (EMC) community. Measuring the emitted fields in the near field of the DUT and transforming this near-field data to the far-field has been mentioned by many before as an interesting alternative pre-compliance measurement method, giving much more insight on the possible root causes of EMC emission problems. Near-field measurement techniques were originally developed for antenna characterization, but later were successfully applied to other areas, among which EMC. The equivalence theorem $[1,2]$ provides a theoretical background for the transformation of near-field data into the far-field. Unfortunately, this theorem requires that the near-field is

Manuscript received August 26, 2014, revised June 8, 2015, accepted July 19, 2015. V. Volski, S. Yan and G. A. E. Vandenbosch are with the Katholieke Universiteit Leuven, ESAT-TELEMIC, Kasteelpark Arenberg 10, 3001 Leuven, Belgium (e-mail: vladimir.volski@esat.kuleuven.be, sen.yan@esat.kuleuven.be, guy.vandenbosch@esat.kuleuven.be). T. Claeys D. Pissoort are with the Flanders' Mechatronics Engineering Center, Faculty of Engineering Technology, University College Katholieke Hogeschool Brugge-Oostende (KHBO), Ostend, Belgium, and also with ESAT-MICAS at the Katholieke Universiteit Leuven, Leuven 3000, Belgium (e-mail: tim.claeys@kuleuven.be, davy.pissoort@kuleuven.be) [This work is performed within the framework of the IWT project NEATH] known on a closed surface fully containing the radiating DUT. For EMC purposes, where near-field scanning is most often applied at the PCB level, a planar scanner is typically used. In that case, the near-field data is limited to a finite rectangular area at a few $\mathrm{mm}$ or $\mathrm{cm}$ above the DUT. Neglecting the field components outside the scan area in this case may lead to huge errors. Most of the existing alternative methods to transform planar near-field data into the far-field [3-9] are based on the introduction of equivalent sources that generate similar near field components inside the scanning area. The types of sources (electric and/or magnetic) depend on the available measured field components and the exact algorithm. Infinitesimal electric and/or magnetic dipoles are very convenient because all field components can be calculated analytically. The general idea actually leads to a classical inverse problem, which in many cases delivers non-unique solutions and involves operations with ill-conditioned matrices. In order to stabilize the solution several remedies already have been proposed, involving special optimizations and/or iterative algorithms, combinations with full wave solvers, etc. Other approaches treat near field data involving plane wave spectra [10]. Although this approach also suffers from the truncation of the scan data, it allows to derive all field components in the spectral domain at different heights. A very good overview of the different methods can be found in $[5,10]$.

The main goal of this paper is to propose a simple and flexible algorithm capable of predicting the level of radiated emissions from a DUT (typically a PCB) based only on planar scan data and topological information about the DUT's dimensions. A first step in this direction was described in [11], where near field electric field measurements in a fully anechoic chamber are dealt with. A similar concept was tested for magnetic field components [12]. In this paper this concept is further worked out in full detail, including new examples, a filtering procedure based on eigenvalues, the analysis of all field components, and the addition of a finite-sized ground plane. The proposed algorithm relies on simple guidelines and well-known matrix operations only. Moreover, in many cases this algorithm can be combined with other methods to improve the accuracy of the existing method. The major difference with the existing approaches described above is the introduction of auxiliary radiating sources that approximate the fields in the plane outside the finite scanning area and not inside the scanning area itself. It will be shown that this technique offers significant advantages. In this paper, electric dipoles are selected as 
auxiliary sources. Their positions and amplitudes are defined in several steps in order to trace all important possible sources of radiation emission. In general, the ideas presented can be used for any set / combination of near field electric / magnetic field components. In this paper we work out the theory for magnetic field components. The magnetic field is widely used in practice for EMI near field scanning [13] as it is less disturbed by the measurement probe compared to the electric field.

\section{THEORY}

\section{A. The Basic Procedure}

Consider a DUT with electric current distribution $\mathbf{J}^{D U T}$ as shown in Fig. 1. In addition, consider a finite planar scanning area $S_{\text {scan }}$ at a given height above the DUT. In what follows, the remaining part of the entire plane at that given height above the DUT but outside of the finite scanning area will be noted as $S_{\text {out }}$.

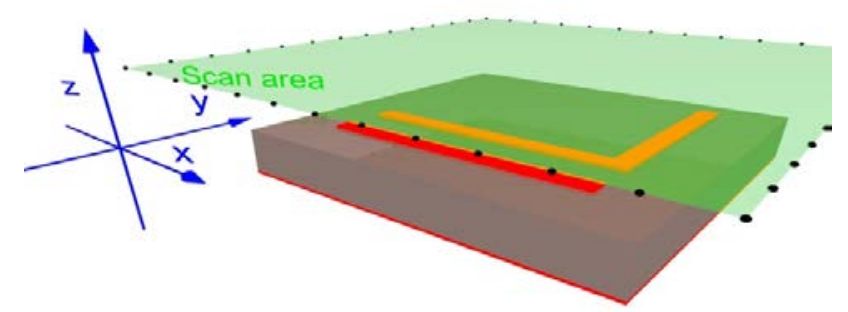

Fig. 1. The topology considered and the edge conditions for the auxiliary dipoles.

The magnetic field components generated by the DUT within and outside the scanning area are $\mathbf{H}_{\text {scan }}$ and $\mathbf{H}_{\text {out }}$, respectively. Using the equivalence principle, the electric field above the scanning plane can be written in terms of the magnetic field in the scanning plane.

$$
\mathbf{E}^{F S}\left(\mathbf{J}^{D U T}\right)=\mathbf{E}^{M C}\left(\mathbf{H}_{\text {scan }}\right)+\mathbf{E}^{M C}\left(\mathbf{H}_{\text {out }}\right)
$$

The superscript FS stands for free space. MC stands for Magnetic Conductor, indicating that the version of the equivalence principle involving only the magnetic field and a PMC (Perfect Magnetic Conductor) is used [1]. The first term on the right hand side is known, since it involves the measured scan area. The second term is unknown since $\mathbf{H}_{\text {out }}$ is not measured. For a planar PMC the magnetic field components can be directly coupled with an equivalent electric current distribution flowing in free space

$$
\begin{aligned}
& \mathbf{J}_{\text {scan }}=2 i_{z} \times \mathbf{H}_{\text {scan }} \\
& \mathbf{E}^{F S}\left(\mathbf{J}_{\text {scan }}\right)=\mathbf{E}^{M C}\left(\mathbf{H}_{\text {scan }}\right)
\end{aligned}
$$

In the remaining part of the paper, the superscripts FS and MC will be omitted.
Now, consider an auxiliary current distribution $\mathbf{J}^{A D}$. These generate known field components everywhere in space, and thus also in the scanning plane, i.e. $\mathbf{H}_{\text {scan }}^{A D}$ and $\mathbf{H}_{\text {out }}^{A D}$. Using the same version of the equivalence principle delivers an equation in which all terms are known.

$$
\mathbf{E}\left(\mathbf{J}^{A D}\right)=\mathbf{E}\left(\mathbf{H}_{\text {scan }}^{A D}\right)+\mathbf{E}\left(\mathbf{H}_{\text {out }}^{A D}\right)
$$

Subtracting (3) from (1) and working out delivers the equation

$$
\begin{aligned}
\mathbf{E}\left(\mathbf{J}^{D U T}\right)=\mathbf{E}\left(\mathbf{J}^{A D}\right) & +\left[\mathbf{E}\left(\mathbf{H}_{\text {scan }}\right)-\mathbf{E}\left(\mathbf{H}_{\text {scan }}^{A D}\right)\right] \\
+ & {\left[\mathbf{E}\left(\mathbf{H}_{\text {out }}\right)-\mathbf{E}\left(\mathbf{H}_{\text {out }}^{A D}\right)\right] }
\end{aligned}
$$

Due to the superposition principle this can be written as

$$
\begin{aligned}
\mathbf{E}\left(\mathbf{J}^{D U T}\right)=\mathbf{E}\left(\mathbf{J}^{A D}\right) & +\mathbf{E}\left(\mathbf{H}_{\text {scan }}-\mathbf{H}_{\text {scan }}^{A D}\right) \\
& +\mathbf{E}\left(\mathbf{H}_{\text {out }}-\mathbf{H}_{\text {out }}^{A D}\right)
\end{aligned}
$$

The core of the proposed procedure consists of determining the auxiliary current distribution in such a way that it generates approximately the same magnetic field in the scanning plane outside the scanning area as the original electric current distribution $\mathbf{J}^{D U T}$ of the DUT, thus

$$
\mathbf{H}_{\text {out }}^{A D} \approx \mathbf{H}_{\text {out }}
$$

The way how to determine the auxiliary current distribution is described in Section II.B. Inserting (6) in (5) delivers

$$
\mathbf{E}\left(\mathbf{J}^{D U T}\right) \approx \mathbf{E}\left(\mathbf{J}^{A D}\right)+\left[\mathbf{E}\left(\mathbf{H}_{\text {scan }}\right)-\mathbf{E}\left(\mathbf{H}_{\text {scan }}^{A D}\right)\right]
$$

In this equation all terms in the right hand side are known. It is absolutely crucial to emphasize that in principle the procedure does not require a good approximation of the field $\mathbf{H}_{\text {scan }}$ by the field $\mathbf{H}_{\text {scan }}^{A D}$ inside the scan area. As soon as the field outside the scan area is sufficiently well approximated, see equation (6), the error in (7) is small, even if $\mathbf{H}_{\text {scan }}$ and $\mathbf{H}_{\text {scan }}^{A D}$ would differ substantially. The reason is that both the actual near field scan and the scan $\mathbf{H}_{\text {scan }}^{A D}$ are taken separately into account in (7). Only outside the scan area, where in general no hotspots or very fast variations of the fields occur, this approximation has to be of sufficient quality.

\section{B. The Auxiliary Sources}

The only basic condition that the auxiliary current distribution has to satisfy is that it approximates the magnetic fields outside the scanning area as good as possible. We will construct this current distribution as a superposition of simple auxiliary sources. A number of questions naturally arise:

1. Which type of auxiliary source do we have to use? 
2. How many do we have to use?

3. Where do we locate them?

4. How do we implement condition (6) concretely?

1. Type: The two most simple and most straightforward auxiliary source types are the infinitesimal electric and infinitesimal magnetic dipoles, further denoted by $\mathrm{J}$ and $\mathrm{M}$. Each of these dipoles can be oriented in three directions (x, y, and $\mathrm{z}$ ), so in total we have 6 elementary dipole types available. Several issues need to be considered. First, whereas the current flowing on a PCB is a smooth surface electric current, the use of infinitesimal dipoles introduces $1 / r^{3}$ and $1 / r^{2}$ false singularities in the near field $[4,8]$. It is important to emphasize that in the proposed technique this is not a fundamental problem. If considered appropriate, the rapid variation of the near field components generated by the dipoles can be smoothened by choosing the distance between the dipoles and the scan area in a proper way. Guidelines used in the method of auxiliary sources [14] can be consulted. Second, it needs to be pointed out that elementary dipoles have intrinsic properties. An electric dipole "flowing" in the horizontal xy plane is not able to produce an $E_{\theta}$ far field component in that plane. This type of component can only be produced by a vertical electric dipole or a horizontal magnetic dipole. Similar considerations are valid for the horizontal magnetic dipole, and for vertical dipoles of both types. This aspect clearly has to be taken into account when choosing the auxiliary sources in order to produce good results. In our procedure, electric dipoles in the three directions are used. This ensures that all far field components can be covered. The selection of electric dipoles is preferable if only magnetic field components are used due to less pronounced source singularities $\left(1 / r^{2}\right)$. Note that combining electric and magnetic scan data, as done in [19, 20], is able to provide a better prediction than only using magnetic scan data. In this case the direct NF FF transformation provides already an acceptable approximation.

2. Number and 3. location: It is well known that the electric currents flowing on the traces of the PCB generate very high magnetic field components in their close vicinity which will be captured by the scan if the scan area is located close enough to the PCB. We assume that both amplitude and phase $\mathrm{H}$-field data are available. First, the scan data of the different field components are combined into one data set as

$$
H=\sqrt{\left|H_{x}\right|^{2}+\left|H_{y}\right|^{2}}
$$

The analysis of the data obtained with (8) reveals several zones with high levels of measured magnetic fields. If the scan area is close enough, these zones cover all dominant sources of unwanted emissions caused by the PCB traces.

Second, all local maxima above some threshold $\Delta_{\max }$ are retrieved. The condition is

$H_{\max }^{\text {local }}>\Delta_{\max }^{\text {scan }} H_{\max }^{\max }$

where $H_{\max }^{\max }$ is the absolute maximum and $H_{\max }^{\text {local }}$ is a local maximum. This condition does not mean that the fields that are weaker are not considered. They are not considered in the part of the procedure to define the auxiliary dipoles. In the complete procedure, all the information of the scan is used, even the very low field levels.

Third, so-called effective zones are formed by keeping all points from the scan data on an upland plateau around a local maximum

$H^{\text {zone }}>\Delta_{\text {zone }}^{\text {scan }} H_{\max }^{\text {local }}$

where $\Delta_{\text {zone }}^{\text {scan }}$ is a zone threshold which depends in general on the distance between the top of the PCB and the position of the scan plane. These two thresholds are chosen based on a compromise. The number of unknowns. as will be explained further, is coupled with the number of zones. A too low threshold $\Delta_{\text {zone }}^{\text {scan }}$ yields very large zones and diminishes the number of unknowns, yielding a less flexible solution. A too high threshold reduces the equivalent area where dipoles are put, resulting in the loss of the contribution from the electric currents actually flowing on the non-selected areas. For a simple pcb with a single trace for example, the width of the obtained zone should be comparable with the width of the trace. If only (10) is used for closely spaced traces, these traces end up into one zone. If it is necessary to keep all traces distinctive, an additional condition is imposed requiring the same direction of the gradient within each zone when moving from the maximum. Such step can be recommended if there is a dominant zone which assimilates all neighboring small zones. This means that a zone containing closely spaced traces can be split up in two zones with two different maxima and a minimum in between. It is preferable that each zone remains within the PCB area. This can be reached by using proper threshold levels in (10) or by just truncating at the edges of the ground plane.

Fourth, auxiliary horizontal dipoles are placed at the top of the PCB $\left(z_{\text {top }}\right)$ where the traces are normally etched as shown in

Fig. 1. Since in normal circumstances, it is sufficient that only a few dipoles cover the selected zone, the placing of these dipoles can be based on a grid with possibly considerably lower resolution than the scan resolution. It is obvious that zonal dipoles mimic rather well the effect of a surface current in test points at the edges of the scan area if the dipole resolution is at least several times smaller than the minimal distance between a dipole and a test point at the edge.

$\mathbf{J}_{H}^{\text {zone }}=i_{z} \times \mathbf{H}^{\text {zone }}$

Within a zone, the amplitudes of the dipoles are the product of a first known factor proportional to the magnetic field observed at the location of the dipole, as described by (11), and one overall unknown amplitude for the whole zone. It is easily seen that in order to have a high contrast in the data, which eases the procedure followed, the scan plane should be as close as possible to the PCB.

Fifth, vertical electric dipoles $J_{z}^{\text {zone }}$ are put in the effective zones. The optimal positions of these dipoles coincide with vias supporting vertical currents and areas where vertical 
polarization currents in the substrate are large. Unfortunately, there is no simple way to extract this information from the near field scan data. Vertical currents flowing on vias are transformed into horizontal currents spreading throughout horizontal traces. These horizontal currents generate distinctive areas with high field levels in the scan data. So, it is logical to assume that the vias are located within the zones already selected. However, there are no direct and obvious parameters indicating how many vias have to be located within each zone. Several $J_{z}^{\text {zone }}$ dipoles are placed inside each zone, again based on an equidistant grid. In contrast to the $J_{H}^{\text {zone }}$ dipoles, which have a fixed amplitudes distribution following the amplitude of the magnetic field, the $J_{z}^{\text {zone }}$ dipoles are chosen independent within each zone, in this way delivering an unknown per vertical dipole. This is because the exact positions of the vias are unknown. As said, the grid used for $J_{H}^{\text {zone }}$ and $J_{z}^{\text {zone }}$ dipoles is based on the grid of the original scan data, but with a lower resolution. The resolution is controlled by defining the minimal distance between two adjacent dipoles $S_{\text {dipole }}$.

4. Implementation of the condition (6): The magnetic field in the horizontal plane outside the scan area can be described as an outgoing wave generated by the real electric current distribution. The auxiliary dipoles grouped in $\mathrm{N}$ zones with each one unknown are also generating a similar outgoing wave. These two outgoing waves are equated in $\mathrm{M}$ test points at the edges of the scan area, resulting in an $\mathrm{M} \mathrm{x} \mathrm{N}$ linear equations system.

$\mathbf{H}\left(\mathbf{J}^{A D}\right)=\mathbf{H}_{\text {scan }}^{\text {edge }}$

The chosen number of test points $M$ should allow a proper interpolation of the variation of the field components at the edges. The obtained system (12) can be solved using the Gauss Legendre least square errors minimum algorithm [15]. The solution in matrix form is

$$
\mathbf{A J}=\mathbf{B} \rightarrow \mathbf{A}^{\mathrm{T}} \mathbf{A} \mathbf{J}=\mathbf{A}^{\mathrm{T}} \mathbf{B} \rightarrow \mathbf{J}=\left(\mathbf{A}^{\mathrm{T}} \mathbf{A}\right)^{-1} \mathbf{A}^{\mathrm{T}} \mathbf{B}
$$

where $\mathbf{A}_{i j}=\mathbf{H}_{i}\left(\mathbf{J}_{j}^{A D}\right)$ is the magnetic field component generated by the $\mathrm{j}$-th group of auxiliary dipoles in the i-th point at the edge and $\mathbf{B}_{i}=\mathbf{H}_{i}^{\text {edge }}$ is the magnetic field component in the same i-th point extracted from the scan data. The inversion of the matrix in (13)

$$
\mathbf{Z}=\mathbf{A}^{\mathbf{T}} \mathbf{A}
$$

can be problematic if it is ill-conditioned. Grouping of the auxiliary dipoles improves the stability of the solution. One way to do this is to use eigenvalue filtering. The eigenvalues and eigenvectors of $\mathrm{Z}$ are calculated and only the eigenvectors with $\lambda_{n} / \lambda_{\max }>\lambda_{\text {threshold }}$ are retained. This means that we use an expansion now in terms of eigenvectors $\mathbf{I}_{n}$.

$$
\mathbf{C}_{n}=\left[\mathbf{I}_{1} \cdots \mathbf{I}_{n}\right]
$$

Note that the total number of retained eigenvectors should be smaller than the number of test points at the edges. In matrix form this transformation results in

$$
\begin{aligned}
& \mathbf{C}_{n}^{T} \mathbf{A}^{\mathbf{T}} \mathbf{A} \mathbf{J}=\mathbf{C}_{n}^{T} \mathbf{A}^{\mathbf{T}} \mathbf{B} \\
& \mathbf{J}=\left(\mathbf{C}_{n}^{T} \mathbf{A}^{\mathbf{T}} \mathbf{A}\right)^{-1} \mathbf{C}_{n}^{T} \mathbf{A}^{\mathrm{T}} \mathbf{B}
\end{aligned}
$$

This regularization technique is similar to the singular value decomposition techniques used in [4]. The choice of the threshold can go along similar lines.

Our method includes the following main steps:

1. the analysis of the scan data (8);

2. the construction of the zones using (9) and (10);

3. the placement of auxiliary dipoles within the zones;

4. the extraction of the test point data from the scan data;

5. the calculation of the field components at the test points;

6. the solution of the system of linear equations (12)-(14);

7. the regularization filtering (16);

8. the calculation of all required field components.

\section{Discussion}

Once the auxiliary dipoles are completely determined, all far field components can be calculated using (7). In order to have a clear physical insight this formula can be rewritten in a slightly different way as

$$
\mathbf{E}\left(\mathbf{J}^{D U T}\right) \approx \mathbf{E}\left(\mathbf{H}_{\text {scan }}\right)+\left[\mathbf{E}\left(\mathbf{J}^{A D}\right)-\mathbf{E}\left(\mathbf{H}_{\text {scan }}^{A D}\right)\right]
$$

The expression in the square brackets is the approximation of the contribution from the missing part of the scan data, i.e. in the outside area

$$
\mathbf{E}\left(\mathbf{H}_{\text {out }}\right) \approx \mathbf{E}\left(\mathbf{H}_{\text {out }}^{A D}\right)=\mathbf{E}\left(\mathbf{J}^{A D}\right)-\mathbf{E}\left(\mathbf{H}_{\text {scan }}^{A D}\right)
$$

The major difference with most existing methods is that the original scan data is not neglected. The presented approach is focused on approximating the missing part of the scan data and actually provides a correction term for the far field pattern. Even if the auxiliary dipoles provide a rather rough approximation of the near field distribution inside the scan area, the improvement of the resulting far field is considerable. This is explained by the ability of the auxiliary $\mathrm{z}$ directed dipoles to reconstruct efficiently slowly decaying $1 / r$ magnetic field components outside the scan area, in contrast with the original (horizontal) equivalent currents (2) which provide only the fast decaying $1 / r^{2}$ components [4, 8].

\section{Ground Plate Currents}

In principle a finite ground plate present at the back of the PCB is automatically taken into account because its contribution to the field is included in the scan data. However, taking it into account more explicitly may improve the accuracy of the results, especially at lower frequencies.

The field at the edges of the scan area can be decomposed in contributions coming from the traces on top of the PCB and the 
ground plate. It is convenient to use image theory and then separately to take into account the diffraction effects caused by the finite size of the ground plate. Since the size of the PCB, and thus also its ground plate may be extremely small compared to the wavelength, a classical Geometrical Theory of Diffraction solution [16] may encounter fundamental problems. However, it is well known that the diffraction caused by the truncation can be expressed in terms of edge currents [17]. We will follow this idea and place extra auxiliary dipoles at the edges of the ground plate. First, the scan data are analyzed in order to retrieve all available field components above the edges of the ground plane resulting in a set of points on a closed contour.

Second, equivalent currents can be introduced as before using (11). It is convenient to express them in terms of components parallel $J_{\mathrm{P}}$ and perpendicular $J_{\perp}$ to the edge and not in terms of $\mathrm{x}$ and $\mathrm{y}$ components

$$
\mathbf{J}_{\text {edge }}=\mathbf{i}_{\mathrm{P}} J_{\mathrm{P}}+\mathbf{i}_{\perp} J_{\perp}
$$

Following the GTD postulates, the scattering field components can be coupled with the incident field components or their derivatives at the diffraction point by diffraction coefficients. Each current component can be artificially coupled to the incident field components. The $J_{\perp}$ component is coupled to the incident electric field components $\left(H_{p}, E_{z}\right)$. According to diffraction theory, this current component should provide the $E_{z}$ dominant contribution in the far field along the scan plane. It is easily seen that in the topology given, this is impossible. A $J$ component never gives a far field $E_{z}$ contribution. Therefore, it is preferable to introduce auxiliary dipoles that are able to mimic correctly the far field scattered components. There are two possibilities $M_{\mathrm{P}}$ or $J_{z}$. The choice of magnetic dipoles is not very convenient if magnetic field scan data are used because of the intrinsically stronger source $1 / r^{3}$ singularities. So we choose $J_{z}$, mimicking the physical effects of scattering. Thus the extra auxiliary sources that mimic the diffraction at the edges of the ground plane have the following components

$$
\mathbf{J}_{\text {edge }}^{A D}=\mathbf{i}_{\mathrm{P}} J_{\mathrm{P}}+\mathbf{i}_{\mathrm{z}} J_{\mathrm{z}}
$$

Note that also these amplitudes are unknowns in the problem. In order to decrease the number of unknowns, all dipoles are grouped in zones around the maxima using two different thresholds $\Delta_{\max }^{\text {edge }}, \Delta_{\text {zone }}^{\text {edge }}$ as before in (9) and (10). All retained dipoles are located at the edges of the ground plane. The full set of auxiliary dipoles includes three contributions

$$
\mathbf{J}_{\text {full }}^{A D}=\mathbf{J}^{A D}+\mathbf{J}_{\text {mirror }}^{A D}+\mathbf{J}_{\text {edge }}^{A D}
$$

The first two terms in (21) are coupled. Only the third term adds unknowns compared to (12)

\section{Numerical results}

\section{A. PCB with multiple traces}

Consider a lossless FR-4 PCB $\left(\varepsilon_{\mathrm{r}}=4.6\right)$ with several traces on top, as shown in Fig. 2. All traces are modeled as PEC. The PCB thickness is $1 \mathrm{~mm}$ and its dimensions are $120 \mathrm{~mm} \times 80$ $\mathrm{mm}$. The magnetic field above the PCB at a height of $2 \mathrm{~mm}$ at $200 \mathrm{MHz}$ was calculated using the FDTD solver that is included in Keysights' 3D EM platform EMPro [18]. The top layer of the PCB comprises a differential pair routed over a slot in the ground plane as well as one single ended trace. The differential traces are excited with $1 \mathrm{~V}$ sources and terminated by $50 \mathrm{Ohm}$. The single-ended trace is excited by a $5 \mathrm{~V}$ source and is open-ended. The scan area is $16 \mathrm{~cm} \mathrm{x} 12 \mathrm{~cm}(0.107 \lambda \mathrm{x}$ $0.08 \lambda$ ) and the used resolution is $0.5 \mathrm{~mm}$ in both directions.

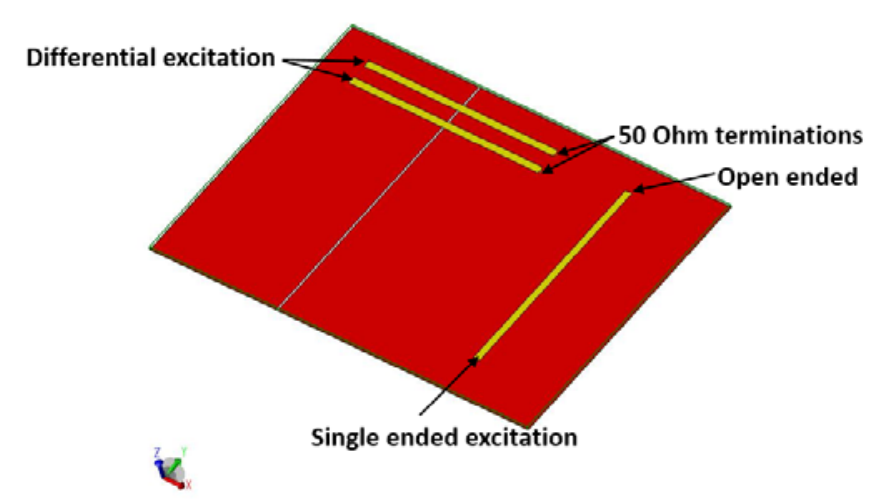

Fig. 2. The screenshot of a PCB test example with several traces on the top.

The combined field components over the scan area $\sqrt{H_{x}^{2}+H_{y}^{2}}$ previous section.

It is important to emphasize that the resolutions used to introduce auxiliary dipoles ( $s_{\text {dipole }}$ ) and to introduce test points at the edges $\left(S_{\text {edge }}\right)$ are much rougher than the resolution of the scan data. The following values were used in the first two examples in this section

$$
\begin{aligned}
& S_{\text {dipole }}=S_{\text {edge }}=0.0025 \lambda=3.75 \mathrm{~mm} \\
& \Delta_{\max }^{\text {scan }}=0.01, \Delta_{\text {zone }}^{\text {scan }}=0.1 \\
& \Delta_{\text {max }}^{g p}=0.01, \Delta_{\text {zone }}^{g p}=0.1
\end{aligned}
$$

These values are chosen in such a way that they allow to locate clearly all main PCB traces. The width of the obtained zones is comparable with the width of the traces and we have single / double rows of dipoles above the traces. The finest resolution is used to locate the maxima of the combined scan data in (8). Within each zone defined by (10) the auxiliary dipoles are placed at the positions indicated by the x-marks in Fig. 3a using the resolution given in (22). This resolution is rougher in comparison with the scan data but is still sufficient to distinguish all main hotspots in Fig. 3.

The total number of auxiliary dipoles is 91 of the $J_{H}^{\text {zone }}$ type and 91 of the $J_{z}^{\text {zone }}$ type, so in total 182. The $91 J_{H}^{\text {zone }}$ dipoles are grouped within 4 zones (yielding 4 unknowns) around the 
retained highest maxima clearly visible in Fig. 3. All $91 J_{z}^{\text {zone }}$ dipoles remain independent (yielding 91 unknowns). So in total there are 95 unknowns. There are 91 x-marks in total in Fig. 3a. Each x-mark covers up to two dipoles, one of type $J_{H}^{\text {zone }}$ and one of type ${ }^{J_{z}^{z o n e}}$, having the same $\mathrm{x}$ and $\mathrm{y}$ position. Each dipole of the $J_{H}^{\text {zone }}$ type has two components ( $\mathrm{x}$ and $\mathrm{y}$ ) and each dipole of the $J_{z}^{\text {zone }}$ type has only one component.

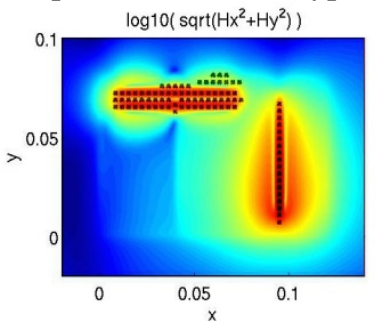

(a)

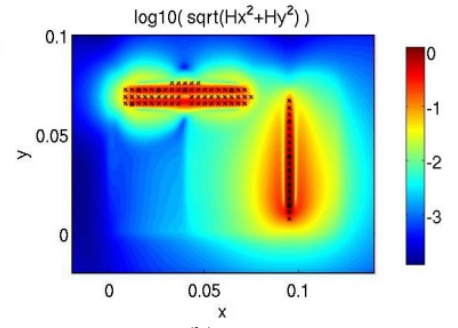

(b)
Fig. 3. Combined scan data and the positions of all original (a) and retained (b) auxiliary dipoles.

The next step consists of the eigenvalue filtering (15). The filtering threshold used in this example is $\lambda_{\text {threshold }}=0.001$. This step reduces drastically the number of dipole groups / unknowns from 95 to 10 and the total number of auxiliary dipoles from 182 to 87 (79 of type $J_{H}^{\text {zone }}$ and 8 of type ${ }_{z}^{\text {zone }}$ ). The remaining dipoles are shown by $\mathrm{x}$-marks in Fig. $3 \mathrm{~b}$.
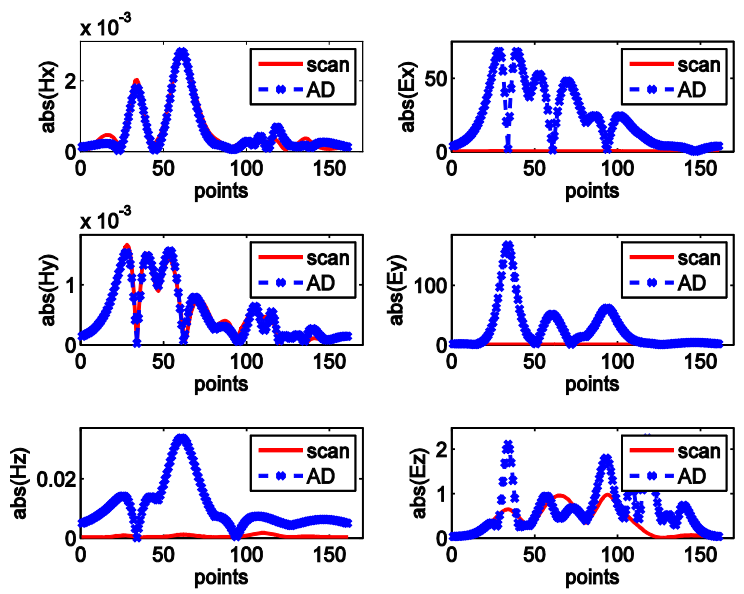

Fig. 4. Comparison of the different field components at the edges of the scan area in the test points.

The condition at the edges of the scan area is imposed only for the $\mathrm{x}$ and $\mathrm{y}$ components of the magnetic field, denoted as $\mathrm{Hx}$ and Hy. It is satisfied in a least square sense because the number of equations is larger than the number of unknowns. All field components at the edges are evaluated or calculated from the scan data and compared with the field components generated by the auxiliary dipoles in Fig. 4 . The edge points on the abscissa are numbered consecutively in the counterclockwise direction, starting from the bottom left corner $\left(\min \left(x_{\text {edge }}^{\text {scan }}\right), \min \left(y_{\text {edge }}^{\text {scan }}\right)\right)$ components are approximated extremely well. The Ez component in the horizontal plane is coupled with $\mathrm{Hx}$ and $\mathrm{Hy}$ and to the average is approximated more or less at the correct level. However, there is an enormous difference for the components $\mathrm{Hz}, \mathrm{Ex}$, and Ey. Since, following the equivalence principle (1), the radiated emission can be calculated accurately if only two components $\mathrm{Hx}$ and $\mathrm{Hy}$ are known, and these two components are approximated well, the crucial question is how this difference will influence the final solution. In this respect it has to be noted that the electric field components lead to equivalent magnetic currents, which are obviously short-circuited when the equivalence principle with the perfect magnetic conductor is used.

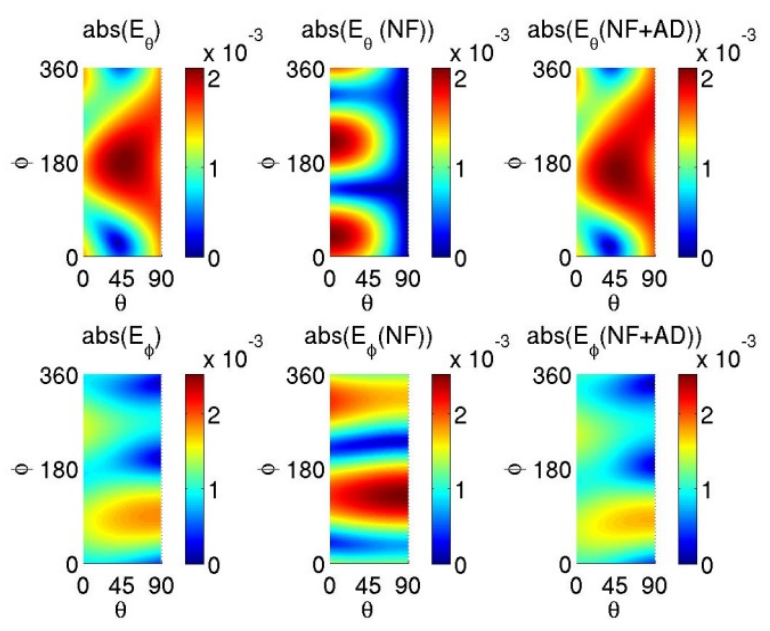

Fig. 5. Reference and approximated far field patterns.

The reference and two calculated far field patterns are plotted in Fig. 5. The colorbar is the same for all sub-figures. The first column corresponds to the reference patterns. The second column shows the results obtained using only the direct transformation of the near field scan data. The last column adds the correction introduced by our approach. It is seen that the direct calculation from the scan data totally fails (second figure in the first row), and that the proposed method allows to restore the far field patterns amazingly well. A comparison with the reference solution for the maximal far field components is presented in Tables 1 and 2. Although in some cases the direct formulation (2) is able to predict rather well the maximal far field level, in all cases it provides very different far field patterns.

Table 1. Maximal far field components for $E_{\theta}$

\begin{tabular}{|l|l|l|l|}
\hline & theta & phi & $\left.\begin{array}{l}\max \left(E_{\theta}\right) \\
{\left[E / E_{r e f}, d B\right.}\end{array}\right]$ \\
\hline reference & $47^{0}$ & $180^{0}$ & $\begin{array}{l}0.00210829 \\
{[0]}\end{array}$ \\
\hline direct & $0^{0}$ & $220^{0}$ & $\begin{array}{l}0.00209389 \\
{[-0.06]}\end{array}$ \\
\hline
\end{tabular}




\begin{tabular}{|l|l|l|l|}
\hline our approach & $50^{0}$ & $160^{0}$ & $\begin{array}{l}0.002101 \\
{[-0.03]}\end{array}$ \\
\hline
\end{tabular}

Table 2. Maximal far field components $E_{\varphi}$

\begin{tabular}{|l|l|l|l|}
\hline & theta & phi & $\left.\begin{array}{l}\max \left(E_{\varphi}\right) \\
E / E_{r e f}, d B\end{array}\right]$ \\
\hline reference & $90^{0}$ & $91^{0}$ & 0.00185449 \\
& & & {$[0]$} \\
\hline direct & $90^{0}$ & $130^{0}$ & 0.00252782 \\
& & & {$[+2.69]$} \\
\hline our approach & $85^{0}$ & $85^{0}$ & 0.00174727 \\
& & & {$[-0.52]$} \\
\hline
\end{tabular}

In order to check the stability of the proposed approach for an increasing initial number of dipoles, a more extensive selection of auxiliary dipoles is used to analyze the same structure. The new set also includes dipoles at the edges of the ground plane and mirror dipoles with respect to the ground plane. The total number of auxiliary dipoles obtained is 542, of which 261 of type $J_{H}^{\text {zone }}$ and 281 of type $J_{z}^{\text {zone }}$, forming 120 groups / unknowns. The dipole positions are shown in Fig. 6a. Each $\mathrm{x}$-mark corresponds with up to 4 different dipoles, including two different types $J_{H}^{\text {zone }}$ and $J_{z}^{\text {zone }}$ and two mirrored dipoles. The $x$-marks at the edges of the ground plane refer to the dipoles associated with $J_{\square}$ and $J_{z}$.

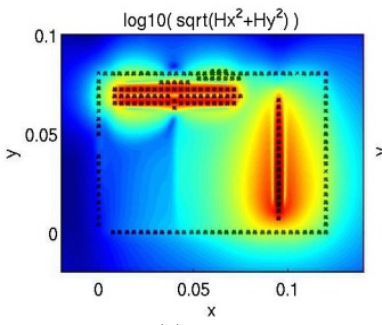

(a)

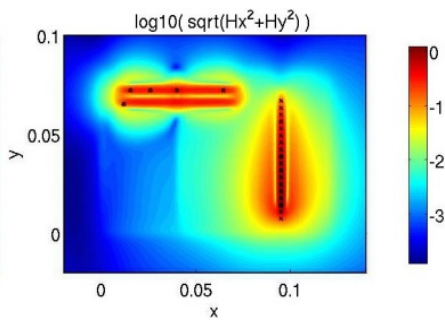

(b)
Fig. 6 Combined scan data and the positions of all original (a) and retained (b) auxiliary dipoles.

As in the previous example, the eigenvalue filtering (14) reduces drastically the number of unknowns (from 120 to 9) and the number of auxiliary dipoles (from 542 to 52) (36 of type $J_{H}^{\text {zone }}$ and 16 of type ${ }_{z}^{\text {zone }}$ ). The remaining dipoles are shown in Fig. 6b. A comparison of Fig. 3b and Fig. 6b shows that the number of retained dipoles decreases more noticeably in Fig. $6 \mathrm{~b}$ after the filtering. Essentially, this is a consequence of the much better approximation of the fields thanks to the use of mirror and/or edge dipoles. They are much more capable to grasp the correct trends in the field behavior. Note that in this case, actually, there are no retained edge dipoles. The reason is that these dipoles will be only retained if diffraction gives a really noticeable contribution to the fields at the edges, which is not the case here.

The comparison of the field components at the edges of the scan area is plotted in Fig. 7. As in the previous case the approximation of $\mathrm{Hx}$ and $\mathrm{Hy}$ is excellent. However, the approximation of the other components has improved considerably, especially Ex and Ey. Now, to the average the order of magnitude of the predicted levels agrees with the reference levels for all these components.
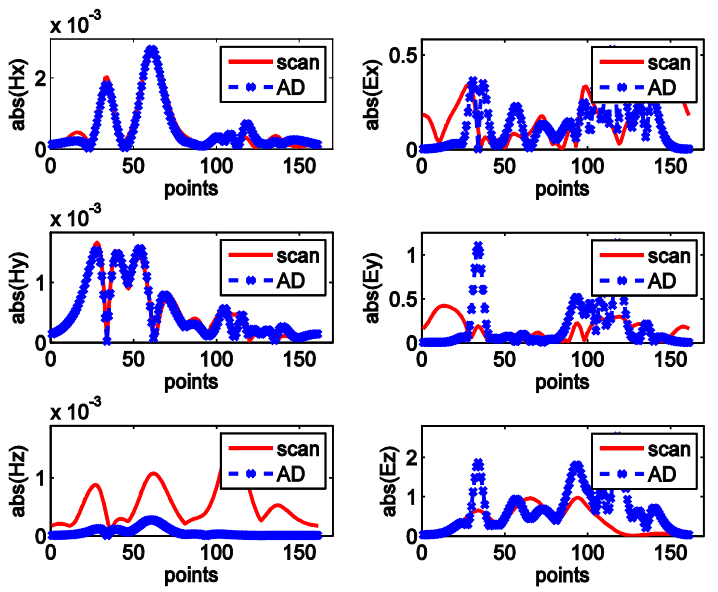

Fig. 7. Comparison of the different field components at the edges of the scan area in the test points.

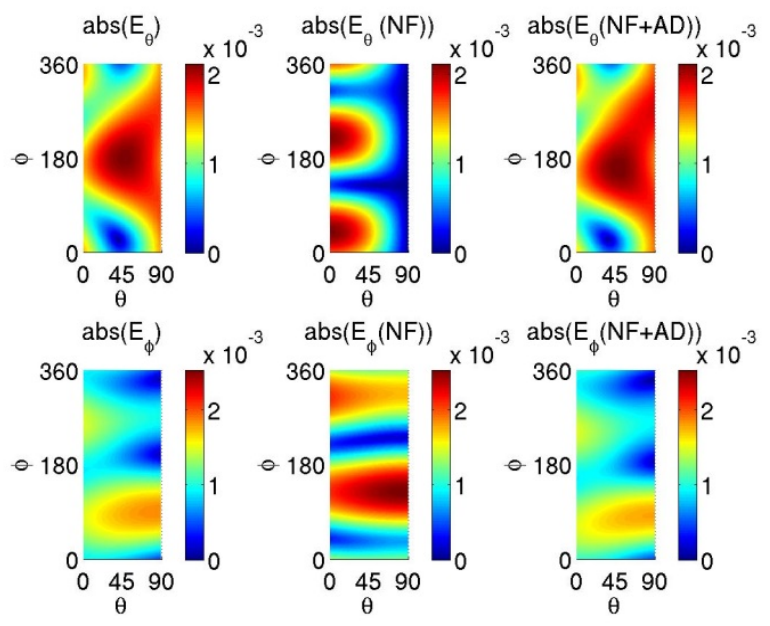

Fig. 8. Reference and calculated far field patterns.

The far field patterns are plotted in Fig. 8. The agreement remains very good despite of the different initial selection of the auxiliary dipoles. A more detailed comparison in the direction of maximum radiation is shown in Tables 3 and 4.

Table 3. Maximal far field components for $E_{\theta}$

\begin{tabular}{|l|l|l|l|}
\hline & theta & phi & $\left.\begin{array}{l}\max \left(E_{\theta}\right) \\
E / E_{\text {ref }}, d B\end{array}\right]$ \\
\hline reference & $47^{0}$ & $180^{0}$ & $\begin{array}{l}0.00210829 \\
{[0]}\end{array}$ \\
\hline direct & $0^{0}$ & $220^{0}$ & $\begin{array}{l}0.00209389 \\
{[-0.06]}\end{array}$ \\
\hline our approach & $45^{0}$ & $160^{0}$ & $\begin{array}{l}0.00211832 \\
{[+0.04]}\end{array}$ \\
\hline
\end{tabular}


Table 4. Maximal far field components for $E_{\varphi}$

\begin{tabular}{|l|l|l|l|}
\hline & theta & phi & $\begin{array}{l}\max \left(E_{\varphi}\right) \\
{\left[\begin{array}{l}E \\
{\left[E_{r e f}, d B\right.}\end{array}\right]}\end{array}$ \\
\hline reference & $90^{0}$ & $91^{0}$ & 0.00185449 \\
\hline direct & $90^{0}$ & $130^{0}$ & 0.00252782 \\
& & & {$[+2.69]$} \\
\hline our approach & $90^{0}$ & $85^{0}$ & 0.00178428 \\
& & & {$[-0.34]$} \\
\hline
\end{tabular}

\section{B. PCB with a double-bended trace}

Consider a second PCB as shown in Fig. 9. All traces are again modelled as PEC while the FR4 substrate is lossy with relative permittivity 4.6 and conductivity $0.0025591 \mathrm{~S} / \mathrm{m}$. The PCB's thickness is $1.6 \mathrm{~mm}$ and its dimensions are $100 \mathrm{~mm} \times 160 \mathrm{~mm}$. The magnetic field above the PCB at a height of $2 \mathrm{~mm}$ at 200 MHz is shown in Fig. 10. The size of the scan area is $13.8 \mathrm{~cm} \mathrm{x}$ $27.8 \mathrm{~cm}(0.092 \lambda \times 0.185 \lambda)$ with a resolution of $1 \mathrm{~mm}$.

The auxiliary dipoles are introduced as in the previous examples using combined field components (8) and (21) with the following parameters

$\Delta_{\max }^{\text {scan }}=0.7, \quad \Delta_{\text {zone }}^{\text {scan }}=0.1$

$\Delta_{\text {max }}^{g p}=0.01, \quad \Delta_{\text {zone }}^{g p}=0.7$

$S_{\text {dipole }}=S_{\text {edge }}=0.0025 \lambda=3.75 \mathrm{~mm}$

$\Delta_{\max }^{\text {scan }}, \Delta_{\text {zone }}^{\text {scan }}$ were selected larger in comparison with the previous example in order to keep all auxiliary dipoles within the PCB ground plate.

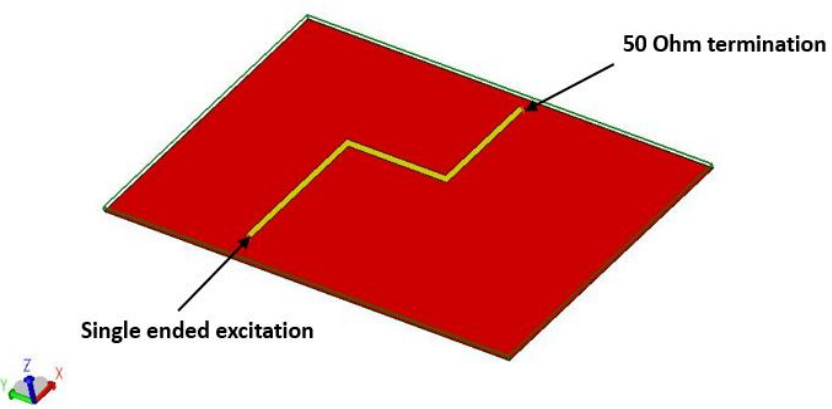

Fig. 9. PCB test example with a double-bended trace routed over the full ground-plane.

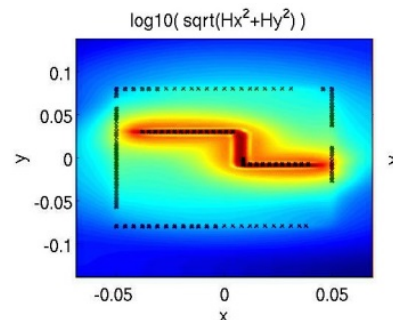

(a)

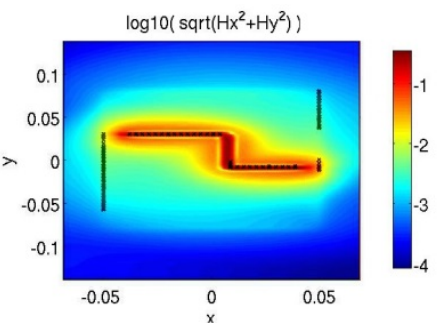

(b)
Fig. 10. Combined scan data and the positions of all original (a) and retained (b) auxiliary dipoles.

The resulting total number of auxiliary dipoles is 225 , forming 48 groups (unknowns), 101 of the type $J_{H}^{\text {zone }}$ and 124 of the type $J_{z}^{\text {zone }}$.

The eigenvalue filtering with $\lambda_{\text {threshold }}=10^{-5}$ reduces the number of unknowns from 48 to 15 and the total number of auxiliary dipoles from 225 to 109 (61 of type ${ }_{H}^{\text {zone }}$ and 48 of type $J_{z}^{\text {zone }}$, as shown in Fig. 10b.

Note that now a few edge dipoles are retained. The comparison of the field components at the edges of the scan area is plotted in Fig. 11. As pointed out already, infinitesimal dipoles can generate false singularities if they are located very close to a test point. In this example, there is a clear sharp peak caused by the dipoles retained at the edges of the ground plate.
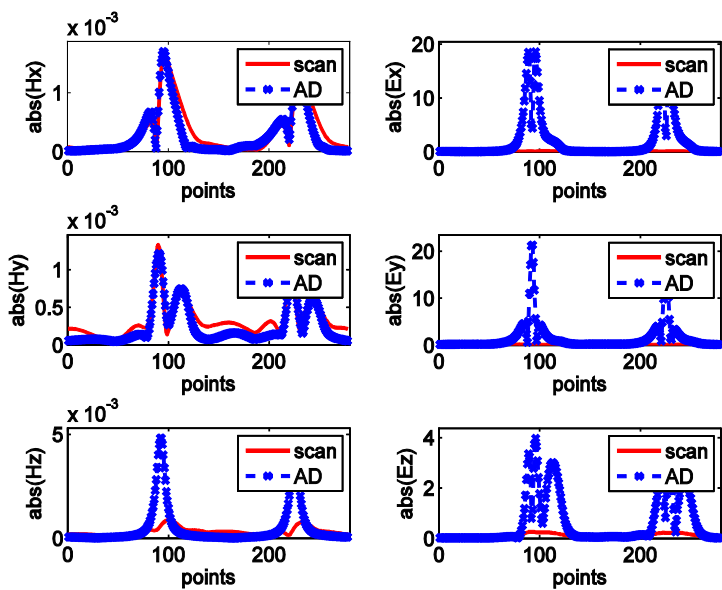

Fig. 11. Comparison of the different field components at the edges of the scan area in the test points. 


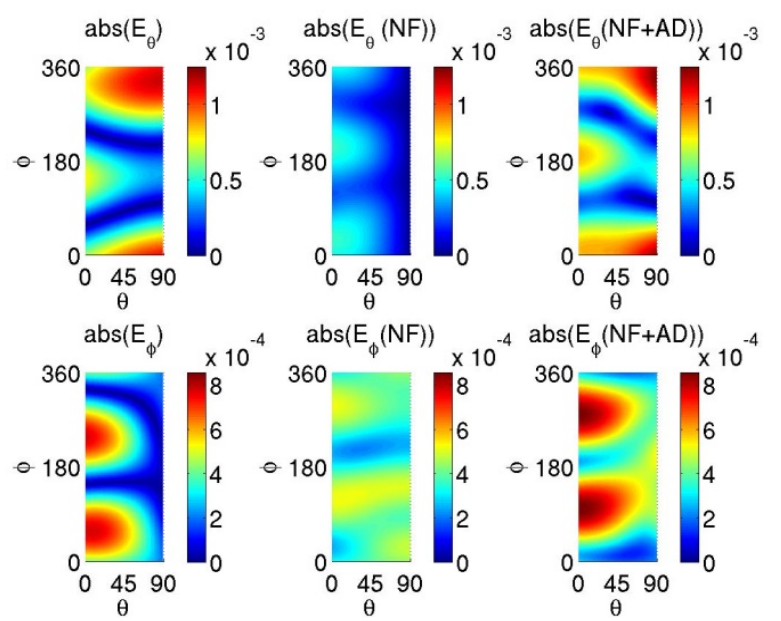

Fig. 12. Reference and calculated far field patterns.

In Fig. 12 and in Tables 5 and 6, the direct transformation gives a lower value of radiation, ca. $6 \mathrm{~dB}$ and $3 \mathrm{~dB}$, respectively. Our method is able to correct this. The improvement of the far field transformation is again very noticeable.

Table 5. Maximal far field components for $E_{\theta}$

\begin{tabular}{|l|l|l|l|}
\hline & Theta & phi & $\begin{array}{l}\max \left(E_{\theta}\right) \\
{\left[\begin{array}{l}E \\
{\left[E_{\text {ref }}, d B\right.}\end{array}\right]}\end{array}$ \\
\hline reference & $90^{0}$ & $335^{0}$ & $\begin{array}{l}0.00114331 \\
{[0]}\end{array}$ \\
\hline direct & $0^{0}$ & $205^{0}$ & $\begin{array}{l}0.00051743 \\
{[-6.88]}\end{array}$ \\
\hline our approach & $90^{0}$ & $340^{0}$ & 0.00124961 \\
& & & {$[+0.77]$} \\
\hline
\end{tabular}

Table 6. Maximal far field components for $E_{\varphi}$

\begin{tabular}{|l|l|l|l|}
\hline & theta & phi & $\max \left(E_{\varphi}\right)$ \\
\hline reference & $6^{0}$ & $57^{0}$ & 0.00077809 \\
& & & {$[0]$} \\
\hline direct & $10^{0}$ & $120^{0}$ & 0.000521247 \\
& & & {$[-3.48]$} \\
\hline our approach & $0^{0}$ & $100^{0}$ & 0.000857667 \\
& & & {$[+0.84]$} \\
\hline
\end{tabular}

The selected zones do not cover the whole PCB. As a consequence the contribution from electric currents actually flowing outside these zones is neglected. Their contribution can be noticeable in some cases. The analysis of Fig. 14 reveals that the agreement between the different field components at the edges is not as good as in the first examples. This is probably due to the more complex shape of the trace, resulting in a more complex field behavior.

\section{Triangular antenna}

Our approach can be also used to estimate the emission from antennas. In our next example we consider an antenna with a triangular patch with a triangular slot and vias, as shown in Fig. 13. It is built using two RO4003 substrates with thickness 1.524 $\mathrm{mm}$. The side lengths of the patch and the ground are $78.51 \mathrm{~mm}$ and $114 \mathrm{~mm}$, respectively. The working frequency is $1.09 \mathrm{GHz}$. The scan data were generated using the CST transient solver with a $1 \mathrm{~mm}$ resolution at the height $7.5 \mathrm{~mm}$ above the patch.

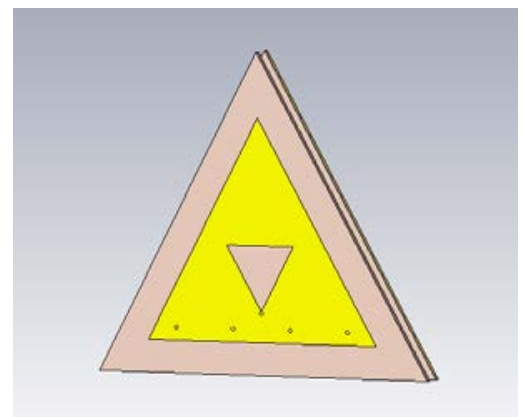

Fig. 13. Triangular antenna with slots and vias.
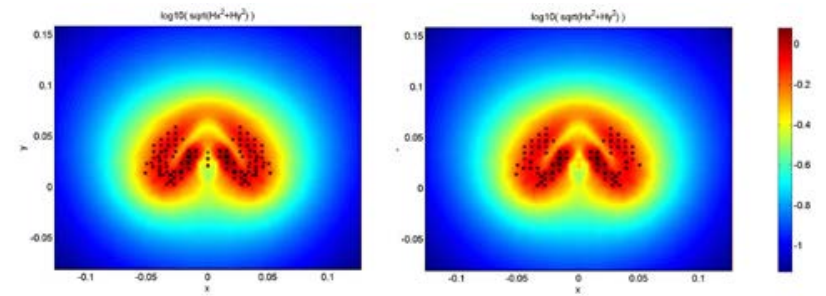

Fig. 14. Combined scan data and the positions of all original (a) and retained (b) auxiliary dipoles.

The positions of the auxiliary dipoles before and after the filtering are shown in Fig. 14. The eigenvalue filtering with $\lambda_{\text {threshold }}=10^{-4}$ reduces the number of unknowns from 36 to 13 and the number of dipoles from 121 to 79 .
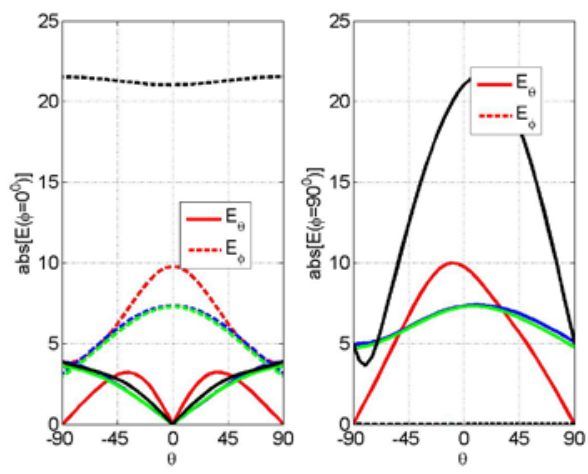

Fig.15. Far field pattern in the plane (a) $\varphi=0^{0}$ (b) $\varphi=90^{\circ}$ (CST - green, direct $\mathrm{NF}$ - red, NF + AD - blue, AD - black).

The far field pattern is plotted in two planes $\varphi=0^{0}$ and $\varphi=90^{\circ}$ in Fig. 15. We have mentioned earlier that our approach does not require that the auxiliary dipoles reconstruct directly the far field pattern. This example is a very convincing illustration of this. Only the combination of the different field contributions in (7) (in blue) is capable to reconstruct accurately the reference far field (in green). The separate field contributions (direct NF 
contribution in red and auxilary dipoles contributioni in black) provide clearly very different patterns.

\section{Circular antenna}

This example involves a patch antenna with multiple slots and vias. The topology generates a quite complex current distribution. The antenna is constructed using two FR4 substrates with thickness $0.8 \mathrm{~mm}$. The circular patch has radius $27.4 \mathrm{~mm}$. It is fed via a coaxial probe at the center and matching is achieved through vias and small circular slots, as shown in Fig. 16. The size of the ground plane is $60 \mathrm{~mm} \times 60 \mathrm{~mm}$. The working frequency is $1.09 \mathrm{GHz}$. The scan data were generated using the CST transient solver with a $1 \mathrm{~mm}$ resolution at the height $7.5 \mathrm{~mm}$ above the patch.

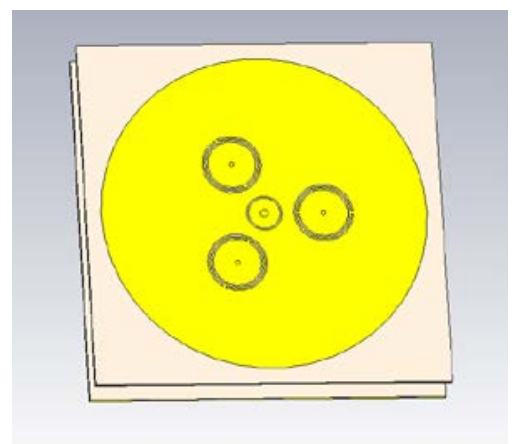

Fig. 16. Circular antenna with slots and vias.

The auxiliary dipoles are introduced using combined field components (8) and (21) with the following parameters

$\Delta_{\max }^{\text {scan }}=0.7, \quad \Delta_{\text {zone }}^{\text {scan }}=0.01$

$S_{\text {dipole }}=0.025 \lambda \approx 6.88 \mathrm{~mm}$

The position of the original dipoles and of the dipoles after the eigenvalue filtering are shown in Fig. 17. The eigenvalue filtering with $\lambda_{\text {threshold }}=10^{-3}$ reduces the number of unknowns (dipole zones) from 155 to 5 and the number of dipoles from 258 to 45.
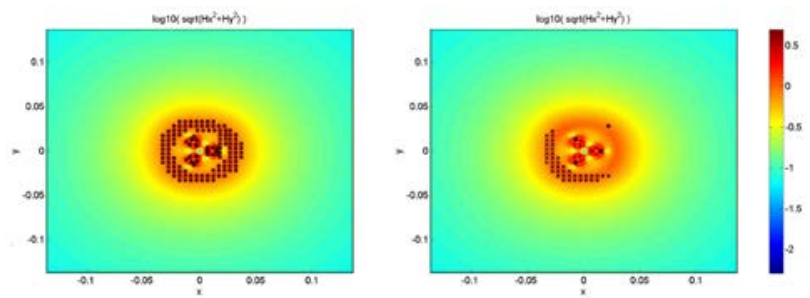

Fig. 17. Combined scan data and the positions of all original (a) and retained (b) auxiliary dipoles.

The far field pattern is plotted in two planes $\varphi=0^{0}$ and $\varphi=90^{\circ}$ in Fig. 18. Different models are plotted using different colors. The reference CST data are plotted in green. The direct NF approach is plotted in red. This antenna behaves like a vertical monopole. It is clearly seen that the direct approach is not capable to describe correctly the $\theta$ component of the electric field in the horizontal plane, leading to a serious deviation (see red line in Fig. 18). The use of the auxiliary dipoles allows to restore the correct far field pattern (see blue line). The separated contributions of the auxiliary is plotted in black. It is clearly seen that in this case the contribution of the auxiliary dipoles is the main contribution.
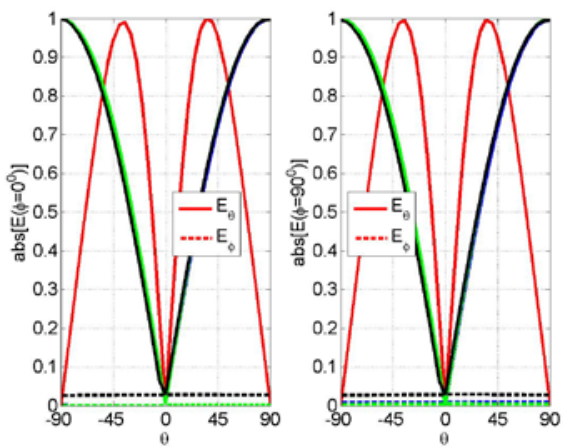

Fig. 18. Far field pattern in the plane (a) $\varphi=0^{0}$ (b) $\varphi=90^{0}$ (CST - green, direct $\mathrm{NF}$ - red, $\mathrm{NF}+\mathrm{AD}$ - blue, $\mathrm{AD}$ - black).

For this antenna also measurements were performed. The near field measurements were performed at the height $7.5 \mathrm{~mm}$ above the patch with a resolution of $2 \mathrm{~mm}$ using a Rohde \& Schwarz ZVB14 vector network analyzer and a SX-R3-1 probe from EMV Langer Technik. The scan data and the positions of the dipoles are shown in Fig. 20. The auxiliary dipoles technique was applied to the measurement data using the following settings

$$
\begin{aligned}
& \Delta_{\max }^{\text {scan }}=0.95, \quad \Delta_{\text {zone }}^{\text {scan }}=0.7 \\
& s_{\text {dipole }}=0.025 \lambda \approx 6.88 \mathrm{~mm}
\end{aligned}
$$

The thresholds were selected higher because the measurement data have a considerably lower contrast. This is mainly due to the lower resolution and the averaging effect of the probe, smoothing very sharp peaks in the field distribution. The thresholds were selected in such a way that the dipoles still cover the main hotspots, resulting in similar dipole distributions in Fig. 17 and Fig. 20. The eigenvalue filtering reduces the number of unknowns (dipole zones) from 53 to 8 and the number of dipoles from 98 to 43 . Various normalized far field patterns are plotted in two planes $\varphi=0^{0}$ and $\varphi=90^{0}$ in Fig. 21 using different colors. The reference CST data are plotted in green. Again, the direct NF approach (red line) suffers from a serious deviation. However our approach (blue line) is capable to restore partially the expected far field pattern, confirming that it can be used in practice.

It has to be emphasized that there is a major issue with this type of measurements. There is a strong coupling of any metallic parts in the measurement set-up with the device, resulting in electric currents on the metallic parts of the measurement device, which results in a huge deformation of the field distribution a bit further (but not that far) away from the device under test. As a consequence, it is very important to replace different metallic parts by non-conductive materials, extensive use of different absorbers, and a special algorithm for probe compensation. The final set-up used to measure the circular antenna is shown in Fig. 19. Although a lot of progress was made, it is still not ideal. A dedicated scanner design would probably yield much better results. However, this issue goes far beyond the scope of this theoretical paper. 


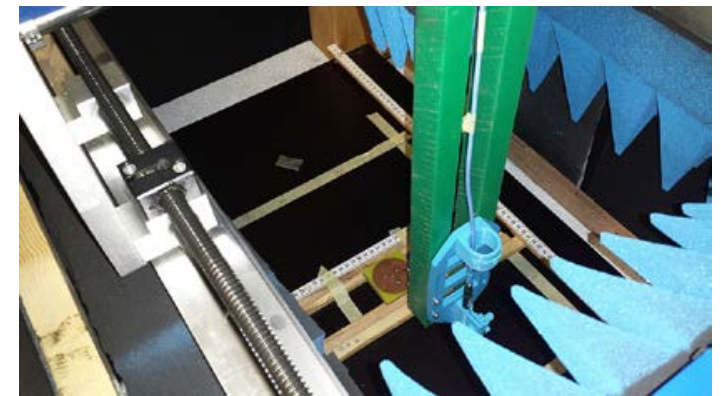

Fig. 19. Near-field measurement set-up with the circular antenna attached.
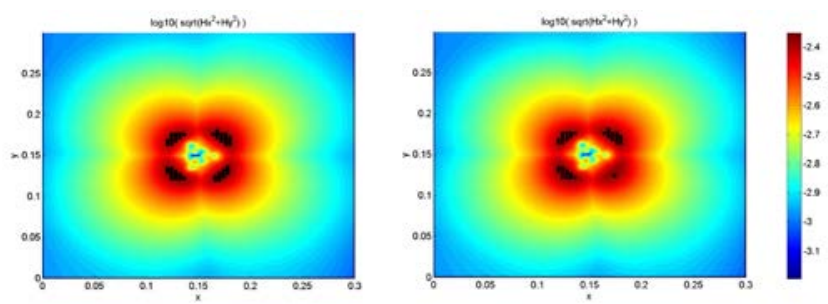

Fig. 20. Combined scan data and the positions of all original (a) and retained (b) auxiliary dipoles.
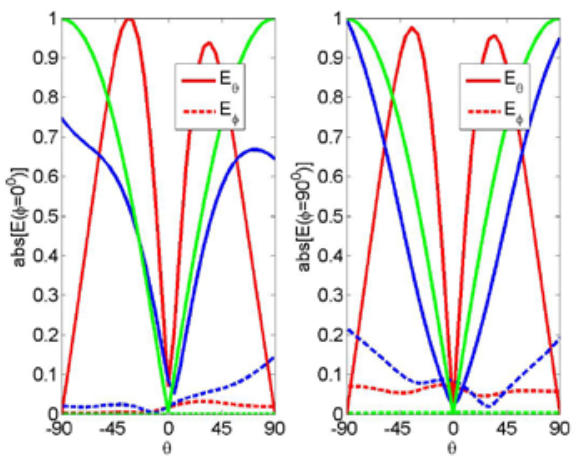

Fig. 21. Normalized far field pattern in the plane (a) $\varphi=0^{0}$ (b) $\varphi=90^{0}$ (CST green, direct NF of measurement scan data - red, NF + AD of measurement scan data - blue).

\section{Conclusion}

A new type of auxiliary sources has been introduced to compensate for the errors in a near to far field transformation due to the finite size of the scanning. The auxiliary sources approximate the field in the missing part of the scanned plane and not inside the scan area itself. It has been shown how with mirrored auxiliary sources and extra auxiliary sources located at the edges of the DUT the influence of a finite ground plane can be accounted for. The proposed idea can be implemented in other methods using auxiliary dipoles. The implementation requires mainly some basic matrix operations involving already calculated data.

On-going research is focusing on the optimal placement and merging of the auxiliary sources, and on the optimal test conditions.

\section{References}

[1] C. Balanis, “Antenna theory: analysis and design”, Wiley, 1997
[2] P. Petre, T.K. Sarkar, "Planar near-field to far field transformation using an equivalent magnetic current approach", IEEE Trans. Antennas Propagation, vol. 40, no.11, pp.1348-1355, Nov. 1992

[3] X. Tong, D.W.P. Thomas, A. Nothofer, P. Sewell, C. Christopoulos, "Modeling Electromagnetic Emissions From Printed Circuit Boards in Closed Environments Using Equivalent Dipoles”, IEEE Transactions on Electromagnetic Compatibility , vol. 52, pp. 462-470, May 2010

[4] Z.W. Yu, J.A. Mix, S. Sajuyigbe, K.P. Slattery, J. Fan, "An Improved Dipole-Moment Model Based on Near-Field Scanning for Characterizing Near-Field Coupling and Far-Field Radiation From an IC” , IEEE Transactions on Electromagnetic Compatibility, vol. 55, No. 1 pp. 97-108, Feb 2013

[5] Y. Alvarez, F. Las-Heras, M.R. Pino, "Reconstruction of equivalent currents distribution over arbitrary three-dimensional surfaces based on integral equation algorithms”, IEEE Trans Antennas Propagation, vol. 55, pp. 3460-3468, Dec 2007

[6] H. Weng, D.G. Beetner, R.E. DuBroff, "Prediction of Radiated Emissions Using Near-Field Measurements”, IEEE Transactions on Electromagnetic Compatibility, Volume: 53 Issue: 4 Pages: 891-899, 2011

[7] Y. Vives-Gilabert, C. Arcambal, A. Louis, P. Eudeline, B. Mazari, "Modeling Magnetic Emissions Combining Image Processing and an Optimization Algorithm", IEEE Transactions on Electromagnetic Compatibility, vol. 51, No.4, Nov. 2009, pp. 909-917

[8] Y. Vives-Gilabert, C. Arcambal, A. Louis, F. de Daran, P. Mazari, "Modeling magnetic Radiations of electronic circuits using near-field scanning method", IEEE Transactions on Electromagnetic Compatibility, vol. 49, No.2, May. 2007, pp. 391-400

[9] P.F. López, C. Arcambal, D. Baudry, S. Verdeyme, and B. Mazari, "Simple Electromagnetic Modeling Procedure: From Near-Field Measurements to Commercial Electromagnetic Simulation Tool”, IEEE Transactions on Instrumentation \& Measurement, Volume 59, Issue 12, Dec 2010, pp.3111 3121

[10] C. Leseigneur, D. Baudry, B. Ravelo, A. Louis, "Near field coupling model PCB and grounded transmission line base on plane wave spectrum”, European Physical Journal Applied Physics, vol. 64, 2013, 11001

[11] G. Vandenbosch, A. Van De Capelle, "Simulation of an EMC reference site in a near field antenna range", EMC Zurich '93 Symposium, pp. 61-66, Zurich, Switserland, 9-11 March 1993, pp.61-66

[12] V. Volski, G. A. E. Vandenbosch, D. Pissoort, "Auxiliary sources for the near to far field transformation of magnetic near field data”, Proc. of the 2014 International Symposium on Electromagnetic Compatibility (EMC Europe 2014), Gothenburg, Sweden, September 1-4, 2014

[13] T. Dorne, F. Vanhee, T. Grenson, D. Pissoort, D. Deschrijver, I. Couckoyt, T. Dhaene, "Optimized Sequential Sampling Algorithm for EMI Near Field Scanning”, Proc. of the 2013 International Symposium on Electromagnetic Compatibility (EMC Europe 2013), Brugge, Belgium, September 2-6, 2013

[14] D.I. Kaklamani, H.T. Anastassiu, "Aspects of the Method of Auxiliary Sources (MAS) in computational electromagnetics", IEEE Antennas \& Propagation Magazine, Volume 44, Issue 3, Jun 2002, pp. 48-64

[15] W.H. Press, S.A. Teukolsky, W.T. Vetterling, B.P. Flannery, "Numerical Recipes 3rd Edition: The Art of Scientific Computing”, Cambridge University Press, 2007

[16] R. G. Kouyoumjian, P. H. Pathak, "A uniform geometrical theory of diffraction for an edge in a perfectly conducting surface," Proc. IEEE, vol. 62, pp. 1448-1461, Nov 1974.

[17] A. Michaeli, "Equivalent Edge Currents For Arbitrary Aspects of Observation”, IEEE Antennas \& Propagation Magazine, Volume 32, Issue 3, 1984, pp. 252-258

[18] Keysight (Agilent Technologies), Agilent EMPro, available from: http://www.keysight.com/

[19] K. Kam, A. Radchenko, D. Pommerenke. "On different methods to combine cable information into near-field data for far-field estimation." Proc. of the IEEE Electromagnetic Compatibility International Symposium, Pittsburgh, USA, August 6-10, 2012

[20] A. Radchenko, J. Zhang, K. Kam, D. Pommerenke, "Numerical evaluation of near-field to far-field transformation robustness for EMC." Proc. of the IEEE Electromagnetic Compatibility International Symposium, Pittsburgh, USA, August 6-10, 2012 


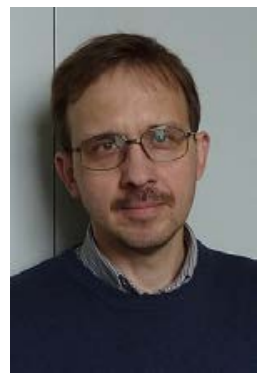

Vladimir Volski graduated from the Moscow Power Engineering Institute, Moscow, Russia, in 1987. In the same year he joined the division "Antennas and Propagation of Radio waves" of the same institute as a researcher. In 1993 he received the degree of Candidate of Science (Ph.D). Since January 1996 he has been a researcher at the ESAT-TELEMIC division of the Katholieke Universiteit Leuven, Belgium. His main research interests include electromagnetic theory, computational electromagnetics, antenna design and measuring of electromagnetic radiation.

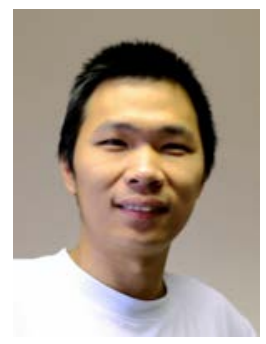

Sen Yan was born in Xianyang, China. He received the Bachelor and Master degrees in Information and Telecommunication engineering from Xi'an Jiaotong University, China, in 2007 and 2010, respectively, and the Ph.D degree in Electrical Engineering from KU Leuven, Belgium in 2015.

$\mathrm{He}$ is currently a postdoctoral researcher at $\mathrm{KU}$ Leuven, focusing on antennas designed based on metamaterials, wearable devices and textile antennas, and microwave chiral meta surfaces.

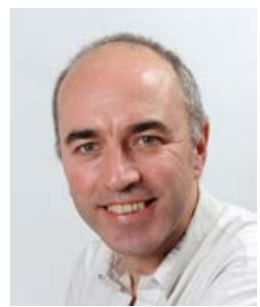

Guy A. E. VANDENBOSCH received the M.S. and Ph.D. degrees in Electrical Engineering from the Katholieke Universiteit Leuven, Leuven, Belgium, in 1985 and 1991, respectively. Since 1993, he has been a Lecturer, and since 2005, a Full Professor at the same university. Guy Vandenbosch has teached or teaches courses on "Electromagnetic Waves", "Antennas", "Electromagnetic Compatibility", "Electrical Engineering, Electronics, and Electrical Energy”, and "Digital Steer- and Measuring Techniques in Physics". His research interests are in the area of electromagnetic theory, computational electromagnetics, planar antennas and circuits, nano-electromagnetics, EM radiation, EMC, and bio-electromagnetics. His work has been published in ca. 225 papers in international journals and has lead to ca. 320 presentations at international conferences. From 2001 to 2007, he was the President of SITEL, the Belgian Society of Engineers in Telecommunication and Electronics. From 2008 - 2014, he was a member of the board of FITCE Belgium, the Belgian branch of the Federation of Telecommunications Engineers of the European Union. In the period 1999-2004, he was vice-chairman, and in the period 2005-2009 secretary of the IEEE Benelux Chapter on Antennas en Propagation. Currently he holds the position of chairman of this Chapter. In the period 2002-2004 he was secretary of the IEEE Benelux Chapter on EMC. In the period 2012-2014 he was secretary of the Belgian National Committee for Radio-electricity (URSI), where he is also in charge of commission E. From September to December 2014, he was a visiting professor at Tsinghua University, Beijing, China.

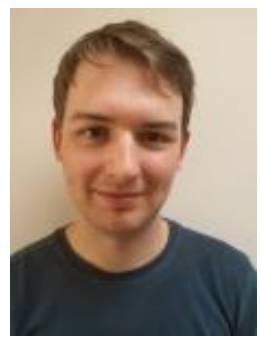

Tim Claeys was born in 1990. He received the M.S. industrial engineering sciences, option electronics, from the University College Katholieke hogeschool Sint-Lieven Gent (KAHO Sint-Lieven), Ghent, Belgium, in 2013. He is currently working towards the Ph.D. degree in electrical engineering from the KU Leuven, Leuven, Belgium. Since 2013, he has been a Research Assistant at the ReMI research group (lab FMEC), KU Leuven Technology campus Ostend, which is a laboratory focusing on global reliability of electronic systems and Electromagnetic Compatibility. His research interests are Near-field scanning, Plane Wave Decomposition and Shielding of gaskets and other materials.

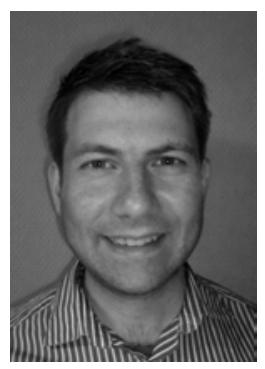

Davy Pissoort (M09, SM13) was born in 1978. He received theM.S. and Ph.D. degrees in electrical engineering from Ghent University, Ghent, Belgium, in 2001 and 2005, respectively. From October 2005 to October 2006, he was a Postdoctoral Researcher Ghent University, Belgium. From November 2006 to July 2009, he was a Research Engineer at the Eesof-EDA Department of Agilent Technolgies in Belgium. Since August 2009, he is Assistant Professor at KU Leuven,
Technology Campus Ostend, Belgium where he is also head of the research group Reliability in Mechatronics and ICT. His current research interests include the development of fast and efficiënt electromagnetic modelling methods for EMC, SI and PI, the development of characterization methods for shielding materials and gaskets, EMI Risk Management as well as the analysis and testing of the mechanical and thermal reliability of electronic modules. He is Senior Member of the IEEE EMC Society and member of the International Steering Committee of EMC Europe. In September 2013 he was vice-chair for the EMC Europe 2013 Symposium in Brugge, Belgium. In August 2015 he was co-chair for the Workshops and Tutorials at the joined IEEE EMC and EMC Europe symposium in Dresden, Germany. 